\title{
Un'analisi dello status della dialisi peritoneale e di alcuni suoi aspetti economici e medici
}

\author{
Giancarlo Ruggieri
}

Nefrologo, Roma

\begin{abstract}
ANALYSIS OF THE STATUS OF PERITONEAL DIALYSIS AND SOME OF ITS ECONOMICAL AND MEDICAL ASPECTS
Abstract. The marginalization of peritoneal dialysis (PD) in Italy has been observed and analyzed more than 10 years ago. The present work reviews this problem concerning PD in Italy as well as worldwide, and evaluates its possible causes on economical and medical bases. The possible relations between the gross domestic product pro-capita (GDP/pC) and the $\%$ of PD have been studied based on the prevalence of PD during the years 2007-2008 in 106 countries, associated to the equivalent GDP/pC data by the World Bank. No direct correlation has been found between GDP/pC and \% of PD, but there was a clear trend in countries with greater incomes to resort less to PD than the countries with lower incomes. This observation has been better defined by relating the GDP/pC to the \% of DP normalized for GDP/pC (PDn): this approach showed an evident and significant difference between countries with greater incomes and those with lower ones, the latter having a DPn share greater than the former ones (among which Italy). In these countries the choice of a dialysis treatment is probably based on different factors than GDP/pC, such as a better reimbursement/cost ratio, and a lesser trust in PD. Some persistent problems concerning the last consideration have been taken into account: the drop out rate, which is still considered in all statistics despite being rather low, and the loss of peritoneal efficiency, due to the reduced, but not yet cut out, damages caused by peritoneal solutions. It is thus possible to believe that the inadequate reimbursements and clinical problems may negatively weigh on the use of PD.
\end{abstract}

Key words: Peritoneal dialysis, Economics, Biocompatibility

Conflict of interest: None.

Financial support: None.

Ricevuto: 12 Giugno, 2013; Accettato: 23 Luglio 2013

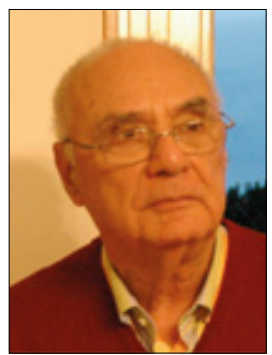

Giancarlo Ruggieri

\section{Premessa}

L'esistenza di una posizione di marginalità nell'uso della dialisi peritoneale (DP) rispetto alle tecniche di dialisi extracorporea (nel loro insieme, HD) si è andata palesando in modo evidente nel corso degli anni, malgrado una sua crescita costante: 1978: 0.15, 1981: 4.99, 1984: 6.3, 1990: 8.14 (dati Censimenti ANED) e 2008: 10.54 (dato SIN RIDT 2008), ovvero 9.6 secondo dati internazionali (vedi in seguito), con una costante forte disomogeneità di prevalenza fra le Regioni italiane. Ciò portò, nel 2001, una rivista italiana a sondare su questo argomento un gruppo di Nefrologi esperti in materia, dedicando a questa indagine un numero monografico (1). Semplificando e citando le più condivise fra le possibili cause ipotizzate per questo fenomeno, esse venivano ricondotte all'eccessivo affidamento in molte Regioni della terapia dialitica a strutture priva- te con il conseguente conflitto di interesse verso una dialisi domiciliare, a una persistente "diffidenza" verso le possibilità della DP come terapia a lungo termine, talora anche laddove si esercitava la DP, e, infine, a una sottovalutazione economica dei rimborsi per la DP, ignorandone i vantaggi economici per la collettività rispetto ai costi dell'HD. A oltre dieci anni da quelle valutazioni, questa elaborazione intende esaminare alcuni aspetti epidemiologici, economici e medici della dialisi peritoneale in periodi recenti.

\section{Epidemiologia della DP}

Il lavoro di Jain et al. (2) costituisce un'importante fonte per lo studio epidemiologico della DP. In esso gli Autori hanno rilevato la prevalenza, assoluta, percentuale e per milione di abitanti, della DP fra il 1997 e il 2008 in 130 Paesi, fornendo i dati di 120 Nazioni per gli anni 2007-2008, divisi per Paesi sviluppati (29) e Paesi in via di sviluppo (91). Lo studio ha anche determinato il trend di utilizzo percentuale della DP fra il 1997 e il 2008 su 67 Paesi in via di sviluppo e 30 svilup- 
pati, evidenziando una perdita dell' $1.38 \%$ nei Paesi in via di sviluppo e del 5.3\% nei Paesi sviluppati, con una prevalenza nel 2008 rispettivamente del $12.4 \%$ e del $15.3 \%$, anche se il trend per milione di abitanti risultava in crescita: da questi dati si è ritenuto di poter affermare la presenza di un andamento ubiquitario in riduzione della DP. La modesta prevalenza della DP sul totale della popolazione in dialisi è stata confermata dal censimento Fresenius 2011 (3), che dà, in un grafico, le prevalenze percentuali della DP in un campione di $15 \mathrm{~Pa}$ esi di varie aree del mondo, selezionati perché titolari delle maggiori prevalenze di soggetti in dialisi: elaborando i dati del grafico, la media della DP risulta essere del $14 \% \pm 14.78$, con una mediana pari al $9.63 \%$ e un coefficiente di variazione 1.056 , dati che dimostrano, oltre alla moderata prevalenza, la grande variabilità d'uso della DP nei 15 Paesi: il range varia dal $61.8 \%$ del Messico allo 0\% dell'Egitto. Nel gruppo sono presenti quattro Paesi europei (Germania, Francia, Italia, Regno Unito), la cui media per la DP è del $9.39 \% \pm 4.24$, con coefficiente di variazione 0.45 , denotante una variabilità medio/ alta fra i quattro Paesi: il dato relativo all'Italia è del $9.6 \%$. Lo stesso censimento dà un valore globale di 2.164 .000 pazienti in dialisi, di cui l' $11 \%$ in DP. Lo status della DP in Italia non è di semplice valutazione, per incompletezze dei dati disponibili dai Registri Regionali così come dai Report SIN RIDT, anche per l'assai frequente esposizione dei dati non con tabelle numeriche ma con grafici, i quali non sempre sono suscettibili di essere adeguatamente trasposti in dati numerici. Un recente lavoro di Marinangeli et al. (4), per conto del Gruppo di Studio della dialisi peritoneale, dà notevoli informazioni sugli anni 2005-2008, fra cui il ricorso alla DP incrementale, $11.92 \%$ dei prevalenti in DP. La prevalenza percentuale della DP calcolata dal gruppo di lavoro per il 2008 è del $16.7 \%$ su
223 Centri tutti praticanti la DP; viene anche segnalata una caduta del $7.63 \%$ dei soggetti in DP fra il 2005 e il 2008, ma, non essendo indicati i prevalenti totali in dialisi nei due anni in tutti Centri con e senza DP, non è possibile valutare l'eventuale caduta percentuale della DP. Il dato 2008 di Marinangeli, riguardante esclusivamente i 223 Centri effettuanti la DP, è, ovviamente, molto superiore al $10.54 \%$ dato dal Report SIN RIDT 2008, che si basa su tutti i Centri dialisi attivi in Italia con o senza DP, da cui, peraltro, mancano i dati di alcune Regioni evidenziate in una mappa d'Italia, verosimilmente tutti quelli dell'Abruzzo e del Molise e parzialmente quelli della Campania: il dato del $10.54 \%$ potrebbe, quindi, essere inesatto per eccesso, tenendo conto che le Regioni carenti non sono importanti utilizzatori della DP mentre hanno un'ampia popolazione in HD. Nell'elaborazione nella Tabella I si è preferito utilizzare i dati ricavati dai Registri Regionali SIN, limitatamente a 10 Regioni, le sole $i$ cui dati risultavano utilizzabili a fine elaborazione.

Con i limiti dovuti all'incompletezza dei dati (10 Regioni su 19), la Tabella I costituisce un confronto dell'utilizzo percentuale della DP fra due periodi successivi, con un intervallo di anni compreso fra 9 e 2 anni, in media $6 \pm 1.9$, con un coefficiente di variazione (CV) di 0.3, quindi considerabile discretamente omogeneo. I periodi di osservazione variano in dipendenza della variabile utilizzabilità dei dati nei vari Registri. Il primo periodo è assunto come base di confronto. Dalla Tabella va rilevato: a) la media delle medie percentuali ottenute sull'uso della DP per il periodo iniziale e per il successivo (media generale) concorda fortemente con i valori dati per l'Italia dai censimenti internazionali ricordati sopra per i periodi considerati e di un altro studio citato qui di seguito, risultati tutti pari al $9.6 \%$ versus il $9.64 \%$ e il $9.42 \%$ delle

TABELLA I - VARIAZIONI PERCENTUALI DI UTILIZZO DELLA DIALISI PERITONEALE FRA DUE DIVERSI PERIODI (ARCO DI TEMPO CONSIDERATO) IN DIECI REGIONI ITALIANE

\begin{tabular}{|c|c|c|c|c|c|c|c|c|c|c|}
\hline \multirow{2}{*}{\multicolumn{2}{|c|}{ Regioni }} & \multicolumn{2}{|c|}{$\begin{array}{l}\text { Arco di tempo } \\
\text { considerato }\end{array}$} & \multicolumn{2}{|c|}{ Variazioni \% } & \multicolumn{5}{|c|}{$\begin{array}{c}\text { Arco di tempo } \\
\text { considerato }\end{array}$} \\
\hline & & 2001 & 2010 & $\pm \%$ & Per anno & Regioni & 2003 & 2007 & $\pm \%$ & Per anno \\
\hline \multirow{2}{*}{\multicolumn{2}{|c|}{ Lombardia }} & $15.9 \%$ & $17.55 \%$ & 1.65 & 0.18 & Sardegna & $6.83 \%$ & $6.36 \%$ & -0.47 & -0.157 \\
\hline & & 2001 & 2008 & & & & 2001 & 2008 & & \\
\hline \multirow{2}{*}{\multicolumn{2}{|c|}{ Lazio }} & $4.4 \%$ & $5.97 \%$ & 1.57 & 0.224 & Sicilia & $3.79 \%$ & $4 \%$ & 0.21 & 0.03 \\
\hline & & 2004 & 2009 & & & & 2004 & 2010 & & \\
\hline \multirow{2}{*}{\multicolumn{2}{|c|}{ Piemonte - V. d'Aosta }} & $16 \%$ & $11.5 \%$ & -4.5 & -1.125 & Friuli Ven. Giulia & $11.4 \%$ & $14.7 \%$ & 3.3 & 0.66 \\
\hline & & 2001 & 2007 & & & & 2007 & 2009 & & \\
\hline \multirow{2}{*}{\multicolumn{2}{|c|}{ Puglia }} & $8 \%$ & $8.12 \%$ & 0.12 & 0.024 & Umbria & $8.7 \%$ & $8.5 \%$ & -0.2 & -0.1 \\
\hline & & 2002 & 2008 & & & & 2001 & 2009 & & \\
\hline \multicolumn{2}{|l|}{ Emilia Romagna } & $8.68 \%$ & $9.68 \%$ & 1 & 0.2 & Calabria & $12.8 \%$ & $7.9 \%$ & -4.9 & -0.54 \\
\hline Medie generali & Medie & 10.59 & 10.56 & -0.03 & -0.0994 & Medie & 8.7 & 8.29 & -0.41 & -0.0214 \\
\hline Periodo $1^{\circ}: 9.64$ & DS & 5.15 & 4.4 & 2.57 & 0.579 & DS & 3.59 & 3.98 & 2.93 & 0.436 \\
\hline Periodo $2^{\circ}: 9.42$ & $\mathrm{CV}$ & 0.486 & 0.417 & -80 & -5.83 & $\mathrm{CV}$ & 0.413 & 0.48 & -7.11 & -20.37 \\
\hline
\end{tabular}

Media della variazione percentuale della Dialisi Peritoneale per anno su tutte le Regioni valutabili: $\mathbf{- 0 . 0 6 0 4 \pm 0 . 4 6 , ~ m e d i a n a ~} 0.027$. 
due medie generali nella Tabella; b) Piemonte, Valle d'Aosta e Calabria presentano le più alte perdite di DP, con un valore medio del 4.7\% mentre Lombardia, Emilia Romagna, Lazio e Friuli Venezia Giulia la incrementano in media dell'1.88\%; c) il risultato finale, in base al confronto delle medie generali $9.64 \%$ versus $9.42 \%$, dà una perdita del $2.28 \%$, con un decremento medio annuo di $-0.0604 \pm 0.46$, come indicato a fine Tabella. Complessivamente, quindi, la prevalenza della DP nelle Regioni valutate appare cambiata modicamente, con un trend molto debole in decremento, che denuncia, piuttosto, una tendenza all'arresto della crescita, come evidenziato dalla mediana delle variazioni percentuali/anno, che è positiva ma pari allo $0.027 \%$.

\section{Aspetti economici}

La dialisi peritoneale e il reddito dei Paesi. È stato ripetutamente asserito che 1'economicità della DP rispetto alle dialisi extracorporee dovrebbe costituire una delle maggiori forze propulsive per la sua diffusione, non solo rispetto alle tecniche HD a costo più elevato, ma anche rispetto all'HD standard, anche se praticata in autoassistenza. Un incremento dei costi si è, peraltro, verificato anche per la DP, nell'APD, per le maggiori complessità tecniche e terapeutiche introdotte, e per ambedue le strategie di DP per l'introduzione di soluzioni dialitiche più complesse, ritenute più efficaci ma mediamente più costose. Malgrado ciò, una letteratura recente ribadisce il vantaggio economico della dialisi peritoneale (5-9): particolare menzione merita un lavoro assai recente di Karopadi et al. (10). Gli Autori, dopo aver fatto un'assai ampia disamina dei lavori concernenti i costi della terapia dialitica nel mondo e dopo averli esaminati per aree geo-politiche, hanno selezionato 46 Paesi comprendenti Paesi sviluppati e non sviluppati, effettuando anche dei controlli mediante questionari in cui mancavano osservazioni recenti, e sono giunti alla conclusione che, nella maggioranza dei Paesi esaminati, il rapporto del costo $\mathrm{HD} / \mathrm{DP}$ dimostrava una capacità di risparmio della $\mathrm{DP}$, con il massimo rapporto a favore della DP a Hong Kong, 2.53 e con quello più sfavorevole in Egitto, 0.22: per l'Italia, hanno evidenziato una prevalenza del $9.6 \%$ e un HD/DP di 1.81 . Assumendo questa maggiore economicità come vera, ci si dovrebbe attendere una qualche correlazione fra la ricchezza di un Paese e la grandezza di adozione della DP, qualsiasi sia l'erogatore economico della terapia (Servizi Sanitari Nazionali, rimborsi da assicurazioni, spesa privata), tenuto conto che il vantaggio del risparmio dovrebbe essere tenuto presente in ogni caso e per chiunque, dato l'elevato costo della terapia dialitica nelle sue varie forme, valutando di pari efficacia e sicurezza DP e HD. Per verificare, almeno in linea generale, l'esistenza di questa relazione, si sono qui utilizzate le pre-

TABELLA II - RAPPORTO FRA PIL/PC E DP. PER L'INTERPRETAZIONE VEDI TESTO

\begin{tabular}{|c|c|c|c|c|c|c|c|c|c|}
\hline \multirow{2}{*}{\multicolumn{3}{|c|}{$\begin{array}{l}\text { Europa } \\
\text { Paesi: } 29\end{array}$}} & \multicolumn{5}{|l|}{ Africa } & & \\
\hline & & & & \multicolumn{2}{|c|}{ Tutti i Paesi: 23} & \multicolumn{2}{|c|}{ Paesi con DP: 9} & & \\
\hline $\mathrm{PIL} / \mathrm{pC}$ & $\mathrm{PIL} / \mathrm{pC}$ & DP $\%$ & Statistiche & $\mathrm{PIL} / \mathrm{pC}$ & DP \% & \multirow{2}{*}{$\begin{array}{l}\text { PIL/pC } \\
4017\end{array}$} & \multirow{5}{*}{\begin{tabular}{|l}
$\mathrm{DP} \%$ \\
13.69 \\
6.3 \\
15.06 \\
1.01
\end{tabular}} & & \\
\hline Media & 34832 & 11.28 & Media & 2994 & 5.36 & & & & \\
\hline Mediana & 33547 & 9.6 & Mediana & 1252 & 0 & 2670 & & & \\
\hline DS & 26324 & 6.03 & DS & 3255 & 11.36 & 4191 & & & \\
\hline Coeff. Variazione & 0.756 & 0.534 & Coeff. Variazione & 1.087 & 2.12 & 1.04 & & & \\
\hline \multicolumn{3}{|l|}{ Correlazione 0.124} & \multicolumn{3}{|l|}{ Correlazione: 0.056} & \multicolumn{2}{|c|}{ Correlazione: -0.169} & & \\
\hline \multicolumn{3}{|l|}{ America del Nord } & \multicolumn{3}{|l|}{ America Centrale } & \multicolumn{4}{|c|}{ America Meridionale } \\
\hline \multicolumn{3}{|l|}{ Paesi considerati: 4} & \multicolumn{3}{|c|}{ Paesi considerati: 11} & \multicolumn{4}{|c|}{ Paesi considerati: 10} \\
\hline Statistiche & $\mathrm{PIL} / \mathrm{pC}$ & DP $\%$ & Statistiche & $\mathrm{PIL} / \mathrm{p}$ & DP \% & \multirow{6}{*}{\multicolumn{2}{|c|}{$\begin{array}{l}\text { Statistiche } \\
\text { Media } \\
\text { Mediana } \\
\text { DS } \\
\text { Coeff. Variazione } \\
\text { Correlazione }-0.164\end{array}$}} & $\mathrm{PIL} / \mathrm{pC}$ & DP $\%$ \\
\hline Media & 31123 & 24.87 & Media & 8701 & 20.24 & & & 5847 & 12.76 \\
\hline Mediana & 34086 & 13.35 & Mediana & 6254 & 12.6 & & & 5780 & 9.8 \\
\hline DS & 17468 & 27.74 & DS & 7593 & 23.15 & & & 3270 & 10.75 \\
\hline Coeff. Variazione & 0.561 & 1.12 & Coeff. Variazione & 0.873 & 1.14 & & & 0.56 & 0.84 \\
\hline Correlazione -0.769 & & & Correlazione -0.21 & & & & & & \\
\hline \multicolumn{3}{|l|}{ Medio Oriente } & \multicolumn{3}{|l|}{ Asia e Oceania } & & & & \\
\hline \multicolumn{3}{|l|}{ Paesi considerati: 12} & \multicolumn{3}{|c|}{ Paesi considerati: 17 - senza DP: 3} & & & & \\
\hline Statistiche & $\mathrm{PIL} / \mathrm{pC}$ & DP \% & Statistiche & $\mathrm{PIL} / \mathrm{p}$ & DP \% & & & & \\
\hline Media & 24629 & 9.18 & Media & 13215 & 16.21 & & & & \\
\hline Mediana & 18155 & 8.3 & Mediana & 3391 & 13.5 & & & & \\
\hline DS & 24052 & 6.3 & DS & 15933 & 19.35 & & & & \\
\hline Coeff. Variazione & 0.98 & 0.69 & Coeff. Variazione & 1.21 & 1.19 & & & & \\
\hline Correlazione -0.838 & & & Correlazione -0.47 & & & & & & \\
\hline
\end{tabular}


valenze percentuali della DP 2007/2008 del già citato lavoro "Global trends in rates of Peritoneal Dialysis", distribuendole secondo le aree geografiche di appartenenza dei Paesi e affiancandole per ogni Paese alla media del prodotto interno lordo pro-capite (PIL/pC) per gli anni 2007/2008, utilizzando i relativi dati on-line della World Bank, che sono espressi in dollari USA (\$). I Paesi considerati sono stati complessivamente 106, suddivisi nelle aree: Europa, Africa, America del Nord, America Centrale, America Meridionale, Medio Oriente, AsiaOceania (queste ultime insieme). Le elaborazioni sono riportate nella Tabella II. Nelle statistiche è riportato il coefficiente di variazione per porre in evidenza il grado di dispersione dei dati: viene espresso come valore percentuale di 1 (ovvero di 100) e una dispersione considerata assai moderata-moderata è, in genere, entro 0.05-0.25.

Osservazioni sulla Tabella II: a) la Tabella dell'Africa appare divisa in due componenti, in quanto dei 23 Paesi africani solo 9 sono risultati utilizzare la DP. Malgrado la differenza di PIL/ pC fra i Paesi africani senza DP e quelli con DP non abbia alcuna significatività statistica, va sottolineato che il $\mathrm{PIL} / \mathrm{pC}$ dei 14 Paesi senza DP è inferiore del $45 \%$ rispetto a quello dei 9 Paesi con DP, quindi probabilmente insufficiente anche per la dialisi più economica, tenendo anche conto che, con ogni probabilità, tutto il materiale avrebbe dovuto essere importato. Malgrado ciò, la differenza di reddito non spiega da sola il non utilizzo della DP, in quanto, fra i Paesi non utilizzanti la DP, vi sono sei Paesi con reddito pari o superiore a quello di altri che effettuano DP. Su quanto espresso nella Tabella si consideri: i) il $\mathrm{PIL} / \mathrm{pC}$ non rappresenta la reale disponibilità di reddito degli abitanti ma è solo una normalizzazione del PIL utile per attuarne più agevolmente il confronto con quello di altri Paesi, visto che, nella realtà, può essere a disposizione in larga parte di uno Stato non erogante assistenza sanitaria o di singole poche persone fisiche o giuridiche; ii) nei Paesi africani con reddito adeguato la mancanza d'uso della DP potrebbe semplicemente essere dovuta al rifiuto di questa terapia da parte della classe medica, fatto ben noto anche in Italia; b) le correlazioni fra PIL/pC e DP \% hanno un valore elevato e negativo per l'America del Nord, il Medio Oriente e l'Asia-Oceania, il che significa che, in questi Paesi, quanto più elevato è il PIL/ $\mathrm{pC}$ tanto più ridotta è la prevalenza percentuale della DP. Queste correlazioni risultano positive ma assai basse per l'Europa e l'Africa, vale a dire che, in queste aree, PIL/pC e DP \% si muoverebbero nello stesso senso, ma il livello di correlazione è troppo basso per avere un reale significato: altrettanto può essere detto per l'Africa che effettua la DP ma con correlazione negativa e per l'America Meridionale e per l'America Centrale. In definitiva, ciò che risulta è che, in Europa, in Africa nel suo complesso e in America Meridionale e Centrale, non è il livello di reddito dei Paesi a condurre la scelta verso la DP. Le regressioni sia lineare che polinomiale cubica dei valori della DP \% di tutti i Paesi considerati che effettuano DP (87 Paesi) sui corrispondenti valori di PIL/pC non hanno correlazione né significatività alcuna, rispettivamente $\mathrm{R}^{2} 0.0 \mathrm{p} 0.971$ e $\mathrm{R}^{2} 0.09 \mathrm{p} 0.863$. I coefficienti di variazione per la prevalenza DP sono molto alti per tutti i Paesi, dal valore minimo di 0.534 (53.4\%) per l'Europa a un massimo di 1.19 (119\%) per l'AsiaOceania, in media $0.951 \pm 0.238$, a dimostrare la forte diversità

TABELLA III - RAPPORTO FRA PIL/PC E DP NORMALIZZATA SUL PIL/PC NELLE DIVERSE AREE GEOGRAFICHE. PER L'INTERPRETAZIONE VEDI TESTO

\begin{tabular}{|c|c|c|c|c|c|c|c|}
\hline \multicolumn{4}{|l|}{ Europa } & \multicolumn{4}{|c|}{ America del Nord } \\
\hline & $\mathrm{PIL} / \mathrm{pC}$ & DP $\%$ & DP \% norm. & & $\mathrm{PIL} / \mathrm{pC}$ & DP \% & DP \% norm. \\
\hline Media & 34832 & 11.28 & 0.758 & Media & 31123 & 24.87 & 1.91 \\
\hline DS & 26324 & 6.03 & 1.06 & DS & 17468 & 27.7 & 3.22 \\
\hline Mediana & 33547 & 9.6 & 0.381 & Mediana & 34086 & 13.35 & 0.381 \\
\hline \multicolumn{4}{|c|}{ America Centrale } & \multicolumn{4}{|c|}{ America Meridionale } \\
\hline & $\mathrm{PIL} / \mathrm{pC}$ & DP \% & DP \% norm. & & $\mathrm{PIL} / \mathrm{pC}$ & DP \% & DP \% norm. \\
\hline Media & 8160 & 24.7 & 6.01 & Media & 5847 & 12.8 & 3.41 \\
\hline DS & 8114 & 23.34 & 6.6 & DS & 3269 & 10.75 & 4.15 \\
\hline Mediana & 5299 & 18.4 & 3.76 & Mediana & 5780 & 9.8 & 1.29 \\
\hline \multicolumn{4}{|c|}{ Africa tutti i Paesi } & \multicolumn{4}{|c|}{ Africa con DP - 9 Paesi } \\
\hline & $\mathrm{PIL} / \mathrm{pC}$ & DP \% & DP \% norm. & & $\mathrm{PIL} / \mathrm{pC}$ & DP \% & DP \% norm. \\
\hline Media & 2994 & 5.36 & 2.63 & Media & 4017 & 13.7 & 6.73 \\
\hline DS & 3255 & 11.4 & 5.6 & DS & 3952 & 15.05 & 7.44 \\
\hline Mediana & 1252 & 0 & 0 & Mediana & 2670 & 6.3 & 2.89 \\
\hline \multicolumn{4}{|c|}{ Medio Oriente } & \multicolumn{4}{|c|}{ Asia e Oceania } \\
\hline & $\mathrm{PIL} / \mathrm{pC}$ & DP \% & DP \% norm. & & $\mathrm{PIL} / \mathrm{pC}$ & DP \% & DP \% norm. \\
\hline Media & 24629 & 9.18 & 1.27 & Media & 15383 & 19.7 & 6.96 \\
\hline DS & 24052 & 6.3 & 2.21 & DS & 16831 & 19.7 & 10.4 \\
\hline Mediana & 18155 & 8.3 & 0.456 & Mediana & 5405 & 15.2 & 2.09 \\
\hline
\end{tabular}


di utilizzo della DP fra i Paesi della stessa area. La variabilità del rapporto $\mathrm{PIL} / \mathrm{pC}$ versus $\mathrm{DP} \%$ nei Paesi a maggiore reddito è effetto dei criteri fortemente diversi con cui questi Paesi adottano la DP, indipendentemente dal reddito, che è comunque sufficientemente alto da consentire loro di scegliere le terapie indipendentemente dal loro costo.

Un utile indice numerico esprimente l'adozione della DP in funzione del PIL/pC può essere ottenuto utilizzando il metodo seguito da Raymond Vanholder et al. (11) per confrontare i rimborsi delle terapie dialitiche in sette differenti Paesi, il quale ha normalizzato i rimborsi sui PIL/pC di ciascun Paese. Similmente, in questa elaborazione la prevalenza percentuale della DP è stata normalizzata sul PIL/pC dei singoli Paesi per singola area secondo la formula: (DP \%/PIL/pC) x 1000. I risultati sono riportati nella Tabella III e sono rappresentati in

TABELLA IV - TARIFFE MINISTERIALI 2013 IN EURO PER SINGOLA SEDUTA - MEDIA DELLE DIVERSE MODALITÀ DI STRATEGIA PER TERAPIA

\begin{tabular}{|l|l|l|l|l|}
\hline \multicolumn{2}{|l|}{} & \multicolumn{2}{|l|}{$\begin{array}{l}\text { Rapporto tariffe } \\
\text { fra terapie 2013 }\end{array}$} & $\begin{array}{l}\text { Rapporto fra costi pubblici } \\
\text { da tecniche DP verso tecni- } \\
\text { che HD - Costi HD = 100 }\end{array}$ \\
\hline HDB & 149.8 & HDB/CAPD & 3.22 & 31.04 \\
\hline HDF & 232.1 & HDF/APD & 4.24 & 23.57 \\
\hline HF & 258.2 & HF/APD & 4.72 & 21.19 \\
\cline { 1 - 2 } CAPD & 46.5 & & & \\
\cline { 1 - 2 } APD & 54.7 & & &
\end{tabular}

breve nella successiva Figura 1.

Il grafico nella Figura 1 evidenzia in modo diretto come i valori più rilevanti di DP normalizzata (DPn) siano ottenuti dai Paesi con PIL/pC minore, il che non significa che questi abbiano una prevalenza numericamente maggiore di DP ma che, in proporzione al loro reddito, vi è maggiore ricorso alla DP. Ciò conferma quanto già rilevato in precedenza e cioè che un reddito nazionale elevato si accompagna a un trend di minore utilizzo della DP. Questo risultato può essere ulteriormente definito in termini numerici. La somma del PIL/pC di tutte le aree considerate è di $126.985 \$$, mentre quella delle aree a maggior reddito è di $94.584 \$(71.33 \%$ del Pil/pC complessivo), a cui corrispondono i valori di DPn più bassi (frecce con linea continua), mentre il $\mathrm{PIL} / \mathrm{pC}$ totale delle aree a minor reddito (frecce con linea tratteggiata) è di $236.401 \$(28.67 \%)$. La somma dei punteggi di DPn è 29.68. di cui i Paesi a maggior reddito generano il $13.3 \%$ e gli altri Paesi il 70.7\%. Le differenze dei PIL/pC e del punteggio di DPn sono ambedue assai significative (rispettivamente, T test 6.18, p 0.003 e $-4.04, \mathrm{p}$ 0.01 ). L'esame della Figura 1 evidenzia anche che i PIL/pC e le rispondenti DPn si dividono in due gruppi in modo netto. La regressione lineare dei valori della DPn di tutti i Paesi considerati che effettuino DP sui rispettivi PIL/pC ha $\mathrm{R} 0.41$, $\mathrm{R}^{2} 0.169$ e p 0.000 , correlazione inesistente fra PIL/pC e DP espressa in valore percentuale, come enunciato in precedenza: effettuando la regressione polinomiale cubica degli stessi dati si ottiene R $0.567, \mathrm{R}^{2} 0.321$ e p 0.000. Osservandone il grafico nella Figura 2 è possibile vedere la conferma dell'esistenza di una zona di brusca transizione fra i redditi minori con elevata DPn e i redditi superiori con bassa DPn, comprovante la presenza di un'area di livello di reddito intermedio, soglia sopra la quale vi è un utilizzo della DP indipendente dal reddito, come appare evidente dalla Figura 2, in cui, nei Paesi a reddito maggiore, vi è un andamento quasi rettilineo del rapporto PIL/

TABELLA V - RAPPORTO FRA I RIMBORSI DI VARIE FORME DI DIALISI IN SETTE PAESI. ELABORATO DALLA TABELLA V IN VANHOLDER R (11)

\begin{tabular}{|l|c|c|c|c|c|c|c|}
\hline & \multicolumn{2}{|c|}{ Belgio } & \multicolumn{2}{c|}{ Germania } & \multicolumn{2}{c|}{ Olanda } & Regno Unito \\
\hline Terapie dialitiche & CAPD vs & APD vs & CAPD vs & APD vs & CAPD vs & APD vs & CAPD vs \\
\hline HD autogestita & 0.943 & 0.943 & 1.595 & 1.595 & 0.675 & 0.675 & 0.675 \\
\hline HD domiciliare & 0.943 & 0.943 & 1.595 & 1.595 & 0.715 & 0.715 & 0.675 \\
\hline CAPD & & 1 & & 1 & & 1 & 0.823 \\
\hline APD & 1 & & 1 & & 1 & & 0.823 \\
\hline HD Ospedaliera & 0.613 & 0.613 & 1.193 & 1.193 & 0.675 & 0.675 & 0.675 \\
\hline & \multicolumn{2}{|c|}{ Francia } & \multicolumn{2}{|c|}{ Stati Uniti } & Ontario, Canada & \\
\hline Terapie dialitiche & CAPD vs & APD vs & CAPD vs & APD vs & CAPD vs & APD vs \\
\hline HD autogestita & 0.79 & 1.017 & 1 & 1 & 1.267 & 1.46 \\
\hline HD domiciliare & 0.88 & 1.133 & 1 & 1 & 1.652 & 1.905 \\
\hline CAPD & \multicolumn{1}{|c|}{1.294} & & 1 & & 1.153 \\
\hline APD & 0.776 & & 1 & & 0.867 & \\
\hline HD ospedaliera & 0.526 & 0.678 & 1 & 1 & 0.854 & 0.984 \\
\hline
\end{tabular}




\begin{tabular}{|c|c|c|c|c|}
\hline \multicolumn{5}{|c|}{ Rispondenza delle DP \% normalizzate sul PIL/pC ai valori del PIL/pC per aree } \\
\hline Aree & DP normal. & Grafico 1 & $\mathrm{PIL} / \mathrm{pC}$ & Aree \\
\hline Asia Oceania & 6,96 & & 34832 & Europa \\
\hline Africa con DP & 6,73 & & 31123 & Nord America \\
\hline Centro America & 6,01 & & 24629 & Medio Oriente \\
\hline Sud America & 3,41 & & 15383 & Asia Oceania \\
\hline Africa tutti i Paesi & 2,63 & & 8160 & Centro America \\
\hline Nord America & 1,91 & & 5847 & Sud America \\
\hline Medio Oriente & 1,27 & & 4017 & Africa con DP \\
\hline Europa & 0,758 & & 2994 & Africa tutti i Paesi \\
\hline
\end{tabular}

Fig. 1 - Andamento della DP normalizzata sul $\mathrm{PIL} / \mathrm{pC}$ in funzione di questo - vedi testo.

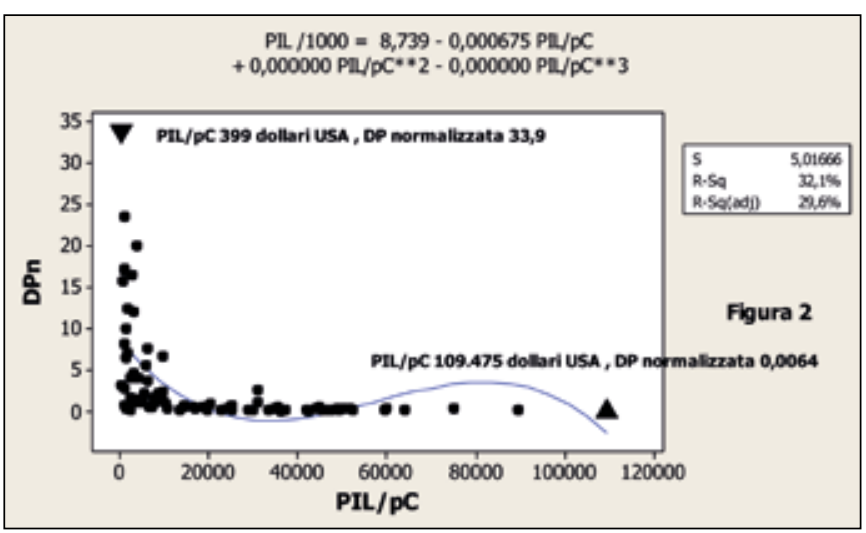

Fig. 2 - Andamento della DP normalizzata su PIL/pC secondo la grandezza del PIL/pC.

pC-DPn, con una tendenza a una riduzione della prevalenza DPn per i redditi estremi.

In questo ambito, l'Italia che, nel 2007-2008, aveva un PIL/ pC di 37.194 \$ e una DP \% di 9.6 presenta una DP normalizzata di 0.26 , dati che rappresentano sui valori complessivi di PIL/pC e di DP normalizzata di tutti i Paesi europei rispettivamente il $3.68 \%$ e l' $1.174 \%$. Sottoponendo a questo conteggio il $16.7 \%$ di DP dato dal lavoro di Marinangeli (4) per 223 Centri dialisi effettuanti DP nel 2008, in base al PIL/ pC italiano del 2008 pari a 38.563 \$, il punteggio della DP normalizzata risulta essere di 0.43 , pari al $2.02 \%$ del punteggio totale di tutti i Paesi europei.

Costi e rimborsi. Il rapporto fra queste due variabili costituisce una delle fondamentali forze di pressione sulla scelta della terapia dialitica in tutti i Paesi, particolarmente dove la terapia sostitutiva è gestita in parte o in toto da strutture profit. La grandezza del rapporto costo/rimborso è regolata dalla reciproca forza di pressione erogatore $\leftrightarrow$ pagatore e dai limiti della disponibilità economica di quest'ultimo: in proposito è stata proposta una formula oltre 10 anni fa (12). Laddove il pagatore è costituito dallo Stato, per la presenza di un Servizio Sanitario Nazionale, si dovrebbe ritenere che questi dovrebbe seguire la via della maggiore economicità a parità di efficacia e sicurezza, ma quest'ultimo giudizio è del
Nefrologo, il quale, per sua convinzione o esperienza, può ritenere non esservi parità fra DP e HD. Rimanendo in tema di valutazione puramente economica, nelle situazioni in cui l'assistenza sanitaria deve soddisfare dei criteri di impresa, la preferenza del medico dovrà, comunque, tenere presente che scegliere fra terapie considerabili di pari efficacia a favore della terapia più prossima a 1 nel rapporto costo/rimborso rispetto a quella con rapporto 1.5 potrebbe essere sottoposto a critica, se non a censura, dal management di impresa, se questa scelta non fosse dovuta a situazioni di terapia obbligata. I vantaggi economici a carattere sociale offerti dalla DP per la sua capacità di abbattere $i$ costi sostenuti dal paziente o dai familiari e di preservare il lavoro nei soggetti capaci di attività lavorativa meglio dell'HD ospedaliera (13) non possono essere presi in considerazione in un'economia di impresa, se non sono stati concretamente inseriti nel calcolo monetario globale del rimborso. Questi vantaggi vengono attualmente ignorati, anche perché lo stesso mondo nefrologico continua a sottolineare l'economicità della DP solo in funzione dei costi diretti di gestione del paziente. La mediocre valutazione economica della DP in Italia è dimostrata dalle tariffe consigliate dal Ministero della Salute per il corrente anno 2013, Supplemento ordinario n. 8 alla Gazzetta Ufficiale, Serie Generale, n. 23, Prestazioni specialistiche ambulatoriali: 28-12013. Il rapporto di queste tariffe fra DP (CAPD e APD) e le altre terapie dialitiche è dato dalla Tabella IV.

Questi rimborsi non sembrano tenere sufficiente conto del costo delle attuali strategie dell'APD e, quindi, non rappresentano un incentivo verso la DP. Al contrario, le tariffe suggerite costituiscono una decisa facilitazione economica per le tecniche a maggiore costo e un poco razionale incoraggiamento ad aumentare i costi dell'insufficienza renale terminale.

Una concreta dimostrazione del reale squilibrio di queste tariffe è evidenziato dai dati della Tabella $\mathrm{V}$, elaborata dal già citato lavoro di Vanholder et al. del 2012 (11), dove viene riportato il rapporto fra i rimborsi delle varie forme di dialisi in sette Paesi. Nella Tabella sono da rilevare in particolare la corretta valutazione del rapporto di rimborso fra l'APD e l'HD ospedaliera (0.984), che tiene conto del costo reale dell'APD, e il rimborso negli Stati Uniti, caratterizzato da 
un'unica tariffa.

Nel valutare gli equilibri dei rimborsi 2013 in Italia, si deve tenere conto di altri due elementi: a) non risulta definito e/o reso noto per tutte le Regioni l'ammontare dei costi accessori prevedibili per la persona in dialisi, come i trasporti con mezzi attrezzati o usuali, l'accompagnamento e gli esami diagnostici di vario tipo non inclusi negli esami standard di controllo, che rimangono in buona parte a carico separato del SSN e il cui conteggio potrebbe chiarire assai meglio il costo totale reale indotto dalle differenti tecniche dialitiche; b) non esiste alcun collegamento del rimborso con il livello di qualità dell'assistenza dialitica erogata e con forme di assistenza medica accessorie e integranti di necessità la dialisi cronica. Nel 2008, il Portogallo, per la crescente difficoltà a fronteggiare la terapia dialitica in una condizione di crisi economica, ha studiato e introdotto una particolare forma di rimborso a quota capitaria, dapprima per gli Ospedali pubblici e poi, dopo lunghe e complesse trattative, anche per le strutture private eroganti emodialisi (14). Questo rimborso non è stabilito per la prestazione dialitica, includente alcuni controlli periodici, ma sul così definito "bundle reimbursement", cioè un rimborso complessivo includente l'erogazione da parte del Centro di Nefrologia della gran parte delle prestazioni necessarie per la persona in dialisi, qui riportate dal testo del lavoro "(1) la terapia dialitica, (2) gli esami di laboratorio e radiologici, (3) tutti i farmaci necessari per il trattamento dell'anemia, della malattia ossea e delle comorbidità nutrizionali e cardiovascolari e (4) la terapia antibiotica endovenosa e, a partire dal 2011, la cura dell'accesso vascolare". Inoltre il pagamento del pieno rimborso è stato legato per legge al raggiungimento di target terapeutici concordati, fra i quali: un $\mathrm{KT} / \mathrm{V} \geq 1.2$, un'emoglobina compresa fra 10 e 13 $\mathrm{g} / \mathrm{dL}$ nel $90 \%$ dei pazienti e una qualità dell'acqua rispondente al 90\% di quanto indicato dalle Linee Guida. Nel 2011, questo sistema è stato adottato anche dagli Stati Uniti, con il riscontro di un netto miglioramento delle condizioni fisiche e dell'outcome dei pazienti (15). Il costo convenuto in Portogallo per il 2011 era di 537,25 euro/settimana. Riducendo convenzionalmente il rimborso ministeriale italiano medio 2013 per le tecniche HD al valore 2011 in base all'indice di inflazione, quindi 200,7 euro, il rimborso/settimana risulta essere di 602,1 euro. Normalizzando ambedue i rimborsi sul $\mathrm{PIL} / \mathrm{pC}$ di Portogallo ed Italia 2011, l'indice portoghese risulta pari a 24.3 e quello italiano a 16.6 con una differenza del $30.9 \%$, differenza nella quale entrano però i costi di una vasta gamma di prestazioni del tutto assenti dalle tariffe ministeriali 2013 e pagate a parte dal SSN, nonché l'aggancio per legge del pieno rimborso all' erogazione di prestazioni adeguate. Questo metodo, finora valutato molto efficace da chi lo pratica, potrebbe/dovrebbe essere adottato anche in Italia, esteso ad ogni forma di terapia dialitica, con sicuro vantaggio di rivalutazione della DP sul piano economico.

\section{Aspetti medici}

Nei primi anni d'uso della DP, il maggiore ostacolo alla sua crescita e la causa maggiore di drop out erano dovute all'incidenza di peritonite, non essendovi, allora, particolari at- tenzioni alla biocompatibilità delle soluzioni dialitiche. Nel 1988, Tarchini et al. (16) riferivano, per il Gruppo di Studio CAPD, che, alla fine del 1985, l'incidenza della peritonite era pari a 18.5 pazienti/mese, che scendeva a $0.50 /$ anno fra il 1985 e il 1989, Lupo et al. (17). Il già citato lavoro di Marinangeli del 2012 dà un rate di peritonite di 0.41 paziente/ mesi nel 2008, un valore che indica certamente un notevole abbattimento di tale rischio, ma che causa tuttora un ben definito numero di drop out della DP: sempre dallo stesso lavoro si evince che per peritonite sono passati all'HD, nel 2008,183 soggetti, pari al $36.75 \%$ di tutti i passaggi per varie cause a HD (498) e al 4.47\% rispetto ai 4094 prevalenti in DP, mentre tutti i pazienti passati a HD per ogni causa rappresentano il $12.16 \%$ dei prevalenti.

Con il passare del tempo e con l'aumento dei pazienti in DP, una differente e crescente attenzione è stata riservata alla scarsa biocompatibilità delle soluzioni di dialisi in uso, tutte basate su glucosio in differenti concentrazioni e a $\mathrm{pH}$ inadeguato, condizioni riconosciute essere la causa di danni peritoneali attraverso molteplici fattori e meccanismi patogenetici, fra cui è rilevante la presenza di prodotti di degradazione del glucosio, capaci di indurre fenomeni a carattere infiammatorio a anche livello sistemico. Inoltre si andavano evidenziando problemi di malnutrizione, dovuti al continuo e forte apporto di glucosio : alterazione del rapporto muscolo/ grasso, fino ad una frequente incidenza di obesità e riduzione del patrimonio di aminoacidi e proteine, dovuta alla permeabilità peritoneale per queste sostanze e ad un'inadeguata loro introduzione per il senso di sazietà legato al continuo input energetico da glucosio. Il danno del peritoneo si palesava attraverso una sua decrescente funzionalità come membrana dializzante, come capacità sia di clearance che di ultrafiltrazione, il che ha spinto l'industria attiva nel campo della dialisi peritoneale a creare nuovi tipi di soluzioni, volti a ridurre se non a sostituire l'uso del glucosio, specialmente nelle sue più elevate concentrazioni, a modificare il $\mathrm{pH}$ delle soluzioni, sostituendo il lattato con lattato-bicarbonato, e a migliorare la condizione nutrizionale usando come agenti osmotici gli aminoacidi. Una delle più importanti innovazioni, risalente ormai a molti anni, è stata la creazione di un polimero del glucosio, l'icodestrina, dissipato assai più lentamente e in minor grado rispetto al glucosio nella cavità peritoneale e, quindi, dotato di un'elevata capacità a mantenere il gradiente osmotico utile per il recupero o l'incremento dell'ultrafiltrazione, evitando o riducendo, in tal modo, l'uso delle soluzioni di glucosio ad alta concentrazione. Molti studi operanti un confronto del livello di biocompatibilità fra precedenti e nuove soluzioni hanno concluso che molto poteva essere atteso da queste innovazioni, avendone accertato un'evidente minore potenzialità lesiva (1822). Per contro, Qi et al. (23), nel 2011, hanno segnalato, in una meta-analisi, di non aver trovato nessuna significativa differenza fra glucosio e icodestrina in termini di mortalità, incidenza di peritonite ed eventi sfavorevoli in genere e altri lavori hanno rilevato manifestazioni di importanti fenomeni infiammatori peritoneali attribuibili all'icodestrina (24-27), la quale è stata giudicata anche non più biocompatibile del glucosio (26) e a cui è stata imputata anche la responsabilità 
di un caso di peritonite sclerosante incapsulante (27). Al di là del variabile giudizio sull'efficacia delle nuove soluzioni, appare, comunque, indubitabile che gli studi che si sono espressi a loro favore hanno, comunque, parlato di riduzione degli effetti lesivi, anche a livello di significatività statistica, ma nessuno di scomparsa o di trascurabile presenza degli effetti biologici negativi evidenziati con le vecchie soluzioni dialitiche. Inserendo le parole chiave "dialysis solutions damage" in PubMed Search e limitando la selezione ai lavori fra il 2013 e il 2002 e ai soli studi su soggetti umani, sono state censite 39 pubblicazioni, 4 riguardanti le soluzioni per dialisi extracorporea e 35 concernenti problemi delle soluzioni per dialisi peritoneale, il che sembra indicare che i problemi di biocompatibilità delle soluzioni per dialisi non sono stati risolti e che sono principalmente le soluzioni per DP ad attirare l'attenzione dei ricercatori, anche per l'ultima introdotta, l'icodestrina, per gli effetti negativi riscontrati sul peritoneo. Una nuova occasione di esposizione all'icodestrina prolungata nel tempo è data dalla crescente adozione della DP incrementale, in cui è prevista la possibilità di un uso di routine dell'icodestrina in uno scambio quotidiano (28). La persistenza dei rischi di sclerosi incapsulante (EPS), segnalata nella recente review del Gruppo di Studio sulla DP a cura di Garosi in Nephromeet (29), sottolinea l'opportunità di ulteriori nuove e migliori soluzioni per DP. Non va dimenticato che la disponibilità delle ormai non più nuove soluzioni per DP con bicarbonato e con icodestrina ha intorno ai 20 anni e, da allora, nulla è stato aggiunto per la pratica della DP dalla ricerca industriale del settore. Va ricordato, infine, che studi sperimentali recenti hanno cercato nuove strade, usando nuove sostanze come fattori osmotici (poliglicerolo iper-ramificato e taurina) $(30,31)$.

\section{Considerazioni finali}

Il già citato lavoro di osservazione a opera di Jain et al. (2), fra gli anni 1997 e 2008, conclude per un trend di riduzione della DP nel mondo. Sono, però, rilevabili, per il 2008, aree con una rilevante prevalenza percentuale della DP: Congo 43.9, Messico 65.8, El Salvador 76.5, Costa Rica 46.9, Colombia 36.6, Hong Kong 79.6 e Nuova Zelanda 36.3, con una media generale del $55.1 \% \pm 18.5$ e un coefficiente di variazione di 0.33 . Si deve, però, notare che, mentre Congo, Messico, El Salvador, Costa Rica e Colombia sono Paesi a basso PIL/pC (media 5.112 $\$ \pm 2956$, coefficiente di variazione 0.58 ), con DP del 53.94 \pm 16.59 , Hong Kong e Nuova Zelanda hanno un PIL/pC medio di $31.071 \$$, con un ricorso medio alla DP del 57.95\%. Tutti i Paesi citati non hanno un Servizio Sanitario Nazionale, eccetto il Messico, la cui copertura sanitaria è, peraltro, parziale: quindi, in tutti questi Paesi, appare ovvio il ricorso alla forma di dialisi complessivamente più economica ma anche assai probabilmente la più o la sola disponibile nei Paesi a basso reddito, dato l'alto costo di creazione e gestione di un Centro di emodialisi e la necessità di importare sia la tecnologia che le soluzioni dialitiche. Dai dati del 2008, è evidente la stridente differenza con l'Europa che, con un PIL/pC di 34.832 \pm 26.324 \$, ha una prevalenza di DP dell'11.28\%, e con gli USA e il Canada, che hanno rispettivamente un PIL/pC di 46.554 \$ e 44.175 $\$$ e una DP $\%$ del $7 \%$ e del $18.5 \%$. Questo è evidenziato bene nella Figura 1, dove si pone in relazione il PIL/pC dei Paesi con la loro DPn: nel grafico l'Europa ha il maggiore $\mathrm{PIL} / \mathrm{pC}$ con la più bassa $\mathrm{DPn}$, seguita in ciò dall'America del Nord, malgrado l'elevata DPn del Messico (6.74). L'alta variabilità della prevalenza percentuale della DP in queste aree dimostra che questo comportamento varia fortemente fra $\mathrm{i}$ Paesi, fermo rimanendo che la più alta DP \% in Europa era pari al non rilevante valore del $23.3 \%$ della Svezia. Si deve sottolineare che, in tutta l'Europa, come negli USA e in Canada, l'assistenza dialitica è curata dallo Stato, che dovrebbe, ovviamente, avere tutto l'interesse a supportare il ricorso a metodiche complessivamente valutabili a più basso costo per vari aspetti: il basso utilizzo della DP in queste aree deve considerarsi secondario sia ai vantaggi economici di impresa per le strutture profit private o pubbliche sia a scelte nefrologiche mediamente non molto favorevoli a questa terapia. Appare evidente la marcata differenza dei rapporti del rimborso per le varie terapie fra l'Italia (Tab. IV) e alcuni Paesi europei (Tab. V), evidentemente ispirate a un'assai differente strategia di valutazione e supporto.

In base a quanto esposto, i motivi fondamentali per un medio-basso se non decrescente ricorso alla dialisi peritoneale in Italia risultano essere:

1) Sul piano economico, un rimborso inadeguato a essere di stimolo per le varie forme di DP, inadeguatezza basata su più errori: a) la totale assenza di valutazione dei risparmi sociali insiti in una terapia che utilizza personale, strutture e organizzazione meno complessi e meno costosi rispetto alle terapie emodialitiche; b) la mancata valutazione monetaria delle capacità di difendere assai meglio la possibilità di lavoro dei soggetti in buone condizioni e ancora in età lavorativa e il lavoro delle persone attive chiamate a occuparsi di inabili o di anziani in dialisi; c) l'evidente sottovalutazione grossolana del costo tecnico complessivo delle differenti terapie peritoneali a fronte della probabile sopravvalutazione dei costi delle tecniche HD, da cui un'insufficiente o liminale copertura delle spese vive per la DP e un inesistente o marginale bilancio economico positivo, con il conseguente pesante squilibrio fra il rimborso della DP e il rimborso delle terapie emodialitiche (di nuovo si confrontino i dati della Tab. IV con quelli della Tab. V). Tali vantaggi, come prima ricordato, possono portare a un conflitto di interessi fra l'utilità di chi paga le spese e l'utilità dell'impresa, quale è oggi anche la struttura pubblica; 2) Sul piano medico, le persistenti problematiche tecnicomediche elencate sopra della DP, per le quali sembra attualmente non esservi una definitiva o, almeno, una migliore soluzione a breve-medio termine, associate, in molti Centri di Nefrologia, a un pre-esistente scarso affidamento verso la DP, non conducono a una naturale diffusione di questa terapia e, associate ai già citati possibili problemi economici, possono spingere piuttosto verso una riduzione del ricorso alla DP, come dimostra l'abbattimento del 7.6\% dei pazienti fra il 2005 e il 2008 nello studio di Marinangeli (4). In proposito, sembra opportuno tenere presenti le osservazioni di Canaud (32), che segnala, nel 2013, come, 
nell'Europa occidentale, l'emofiltrazione on-line abbia superato in prevalenza la dialisi peritoneale e costituisca la seconda terapia più utilizzata dopo l'HD standard.

Sembrerebbe, quindi, utile, per sollecitare una ripresa della DP, un intervento su tre fronti:

1) Economico, operando una concorde azione di pressione sulle autorità sanitarie ed economiche nazionali e regionali per una rivisitazione complessiva dei rimborsi della DP, basata, peraltro, su robuste e non contestabili basi statistiche di calcolo dei costi complessivi DP e HD, diretti terapeutici (inclusi i costi dei farmaci più utilizzati nelle due forme di terapia), diagnostici, indotti (trasporti, rimborsi, sussidi) e, anche, indiretti (spese sostenute da pazienti e familiari), aggiornando, a oggi, in proposito, quanto già valutato in passato (12). Come detto sopra, si può ritenere che molto gioverebbe alla DP un sistema "bundle reimbursement", se basato sulla dimostrazione di variabili di costo e vantaggio di questa terapia migliori rispetto a quelle delle terapie HD; 2) Conoscitivo: indagine sulla valutazione della DP sotto $i$ suoi molteplici aspetti economici, organizzativi e medici da parte del mondo nefrologico, effettuata mediante un questionario in due versioni, rivolto ai Centri utilizzanti o non utilizzanti la dialisi peritoneale;

3) Propulsivo: indagini conoscitive su quanto si stia facendo o si intenda fare nel campo farmaceutico industriale su ulteriori miglioramenti delle soluzioni dialitiche per la DP, sollecitando e supportando fortemente nuove ricerche e investimenti mediante contatti diretti ad alto livello fra la SIN e l'industria interessata e inducendo sensibilizzazioni di opinione in materia, finalizzando a essa meeting nazionali che coinvolgano altre specializzazioni e la medicina del territorio.

\section{Riassunto}

Oltre dieci anni fa venne osservata e analizzata la marginalità in Italia della dialisi peritoneale (DP). Questo lavoro rivisita questo problema della DP in Italia e nel mondo, valutandone le possibili cause su basi economiche e mediche. Sono state studiate le possibili relazioni esistenti fra prodotto interno loro pro-capite (PIL/pC) e DP \%, utilizzando i dati di prevalenza della DP 2007-2008 di $106 \mathrm{~Pa}-$ esi e associando a tali dati quelli dei corrispondenti PIL/ $\mathrm{pC}$ della World Bank. Non è risultata nessuna correlazione diretta fra $\mathrm{PIL} / \mathrm{pC}$ e $\mathrm{DP} \%$, ma è risultata un'evidente tendenza dei Paesi con reddito maggiore a ricorrere meno alla DP rispetto ai Paesi con reddito minore. Ciò è stato definito più chiaramente rapportando il $\mathrm{PIL} / \mathrm{pC}$ con la $\mathrm{DP}$ $\%$ normalizzata sul reddito (DPn): questo approccio ha dimostrato un'evidente e significativa differenza fra Paesi con maggiore e minore $\mathrm{PIL} / \mathrm{pC}$, questi ultimi avendo una quota di DPn assai più elevata rispetto ai primi (fra cui l'Italia), in cui la scelta dialitica è apparsa chiaramente basata su altri fattori che non il $\mathrm{PIL} / \mathrm{pC}$, assai presumibilmente su un miglior rapporto rimborso/costo e su un minore affidamento verso la DP. Per quest'ultimo aspetto sono stati presi in considerazione dei problemi persistenti nell'esercizio della DP, il drop out per peritonite, che, anche se molto ridotto, merita ancora una valutazione in tutte le statistiche, e la perdita di efficacia peritoneale, dovuta ai perduranti danni legati alle soluzioni di dialisi, ridotti ma non ancora aboliti: si deve ritenere che rimborsi inadeguati e problemi clinici pesino negativamente sul trend della diffusione della DP.

Parole chiave: Dialisi peritoneale, Economia, Biocompatibilità

Dichiarazione di conflitto di interessi: L'Autore dichiara di non avere conflitto di interessi.

Contributi economici agli Autori: L'Autore dichiara di non aver ricevuto per la preparazione dell'articolo supporti economici o benefici di alcun genere né sollecitazioni da alcuna persona fisica o giuridica.

\author{
Abbreviazioni: \\ DP: dialisi peritoneale \\ CAPD: dialisi peritoneale ambulatoriale continua \\ APD: tutte le tecniche di dialisi peritoneale automatizzata \\ HD: tutte le tecniche di dialisi extracorporea \\ HDB: emodialisi in bicarbonato \\ HDF: emodiafiltrazione \\ HF: emofiltrazione \\ $\mathrm{PIL} / \mathrm{pC}$ : prodotto interno lordo pro-capite \\ DPn: prevalenza percentuale di dialisi peritoneale normaliz- \\ zata sul PIL/pC
}

\author{
Indirizzo degli Autori: \\ Dr. Giancarlo Ruggieri \\ Via degli Estensi 91 \\ 00164 Roma \\ giancarlo.ruggieri@mclink.net
}




\section{Bibliografia}

1. Dialisi peritoneale: dialisi marginale? Giornale di Tecniche Nefrologiche e Dialitiche 2001; 13 (Suppl. 1): 1-66.

2. Jain AK, Blake P, Cordy P, Garg AX. Global Trends in Rates of Peritoneal Dialysis. J Am Soc Nephrol 2012; 23 (3): 533-44.

3. Fresenius Medical Care. ESRD Patients in 2011 A Global Perspective; www.vision-fmc.com/.../ESRD_Patients_in_2011.

4. Marinangeli G, Cabiddu G, Neri L,Viglino G,Russo R, Teatini UOld and new perspectives on peritoneal dialysis in Italy emerging from the Peritoneal Dialysis Study Group Census. Perit Dial Int 2012; 32 (5): 558-65.

5. Tediosi F, Bertolini G, Parazzini F, Mecca G, Garattini L. Cost analysis of dialysis modalities in Italy. Health Serv Manage Res 2001; 14 (1): 9-17.

6. de Abreu MM, Walker DR, Sesso RC, Ferraz MB. A cost evaluation of Peritoneal Dialysis and Hemodialisys in the treatment of End Stage Renal Disease in Sao Paulo, Brazil. Perit Dial Int 2013; 33 (3): 304-15.

7. Chanliau J, Kessler M. [Peritoneal dialysis for ESRD: financial aspects]. [Article in French]. Nephrol Ther 2011; 7: 32-7.

8. Arrieta J, Rodriguez-Carmona A, Remon C, et al. Peritoneal dialysis is the most-effective alternative for economic sustainability of dialysis treatment. Nefrologia 2011; 31: 505-13.

9. Sharif A, Baboolal K. Update on dialysis economics in the UK in PD in the United Kingdom. Perit Dial Int 2009; 31 (Suppl. 2): $558-62$.

10. Karopadi AN, Mason G, Rettore E, Ronco C. Cost of peritoneal dialysis and haemodialysis across the world. Nephrol Dial Transplant 2013.

11. Vanholder R, Davenport A, Hannedouche T, et al. Reimbursement of Dialysis: a Comparison of Seven Countries. J Am Soc Nephrol 2012; 23: 1291-8.

12. Ruggieri G. Marginalità della Dialisi Peritoneale: probabile frutto di equivoci economici, problemi tecnici ed errori di utilizzo. Giornale di Tecniche Nefrologiche e Dialitiche 2001; XIII (Suppl. 1): 45-6.

13. Ruggieri G, Brunori G, De Vecchi A, et al. Il costo inapparente privato e sociale indotto dalla Dialisi Cronica. Giorn It Nefrol 1996; 13 (Suppl. 8): 1-136.

14. Ponce P, Marcelli D, Guerreiro A, et al. Converting to a capitation system for dialysis payment: the Portuguese experience. Blood Purif 2012; 34: 313-24.

15. Maddux FW. Impact of the bundled of the End-Stage Renal Disease payment system on patient care. Blood Purif 2012; 33: 107-11.

16. Tarchini R, Segoloni GP, Gentile MG, et al. Long term results of CAPD in Italy: a report from Italian CAPD Study Group. Clin Nephrol 1988; 30 (Suppl. 1): 68-70.

17. Lupo A, Tarchini R, Cancarini G, et al. Long term outcome in continuous ambulatory peritoneal dialysis a 10 -years-survey by the Italian Cooperative Peritoneal Dialysis Study Group. Am J Kidney Dis 1994: 24: 826-37.

18. Feriani M. Biocompatible fluids for peritoneal dialysis: do they have a clinical impact? G Ital Nefrol 2007; 24: 320-6.

19. Ahmad M, Shah H, Pliakogiannis T, Oreopoulos DG. Prevention of membrane damage in patients on peritoneal dialysis with the new peritoneal dialysis solutions. Int Urol Nephrol 2007; 39: 299-312.

20. Krishnan M, Tam P, Wu G, Breborowicz A, Oreopoulos DG. Glucose degradation Product's (GDP's) and peritoneal changes in patients on chronic peritoneal dialysis: will new peritoneal dialysis solutions prevent these changes? Int Urol Nephrol 2005; 37: 409-18.

21. Ito T, Yorioka N, Kyuden Y, et al. Effect of glucose polymer on intercellular junctions of cultured peritoneal human mesothelial cells. Nephron Clin Pract 2003; 93: 97-105.

22. Mistry CD, Gokal R. The use of glucose polymer (Icodextrin) in peritoneal dialysis: an overview. Perit Dial Int 1994; 14 (Suppl. 3): $158-61$.

23. Qi H, Xu C, Yan H, Ma J. Comparison of icodextrin and glucose solutions for long-dwell exchange in peritoneal dialysis: a meta-analysis of controlled randomized trials. Perit Dial Int 2011; 31: 179-88.

24. Moriishi M, Kawanishi H. Icodextrin and peritoneal inflammation. Perit Dial Int 2008; 28 (Suppl. 3): 96-100.

25. Moriishi M, Kawanishi H, Tsuchiya S. Impact on peritoneal membrane of the use of icodextrin-based dialysis solution in peritoneal dialysis patients. Adv Perit Dial 2006; 22: 24-8.

26. Gotloib L. The mesothelium under the siege of dialysis solutions: old glucose, new glucose, and glucose-free osmotic agents. Adv Perit Dial 2009; 25: 6-10.

27. Kawanishi H, Shintaku S, Shishida M, Morrishi M, Tsuchiya S, Dohi K. A case of encapsulating peritoneal sclerosis suspected to result from the use of icodextrin peritoneal solutions. Adv Perit Dial 2009; 25: 45-9.

28. Viglino G, Neri L, Barbieri S. Incremental peritoneal dialysis: effects on the choice of dialysis of modality, residual renal function and adequacy. Kidney Int Suppl 2008; 108: 52-5.

29. Garosi G. SIN GDS Dialisi Peritoneale: Peritonite Sclerosante Incapsulante. Nephromeet 2013; 04-21; www.nephromeet/com.

30. Mendelson AA, Guan Q, Chafeeva I, et al. Hyperbranched polyglicerol is an efficacious and biocompatible novel osmotic agent in a rodent model of peritoneal dialysis. Perit Dial Int 2013; 33: 15-27.

31. Nishimura H, Ikehara O, Naito T, Higuchi C, Sanaka T. Evaluation of taurine as an osmotic agent for peritoneal dialisi solution. Perit Dial Int 2009; 29: 204-16.

32. Canaud B, Bowry SK. Emerging clinical evidence on On-Line Hemodiafiltration: does volume of ultrafiltration matter? Blod Purif 2013; 35: 55-62. 